\title{
Three-dimensional evaluation of the maxillary effects of two orthopaedic protocols for the treatment of Class III malocclusion: A prospective study
}

\author{
Brittany Fischer $^{1}$ | Caterina Masucci ${ }^{2,3}$ | Antonio Ruellas ${ }^{4,5}$ | Lucia Cevidanes ${ }^{5}$ | \\ Veronica Giuntini $^{3}$ | Michele Nieri ${ }^{3}$ | Cosimo Nardi ${ }^{6}$ | Lorenzo Franchi ${ }^{3,7}$ (D) | \\ James A. McNamara Jr ${ }^{5,8,9}$ | Efisio Defraia ${ }^{3}$
}

${ }^{1}$ Private Practice Orthodontist, San Diego, California

${ }^{2}$ Sous-section Orthopédie Dento-Faciale, Faculté de Chirurgie dentaire,

Université de Nice Sophia Antipolis, Nice, France

${ }^{3}$ Department of Surgery and Translational Medicine, Orthodontics, University of

Florence, Florence, Italy

${ }^{4}$ Department of Orthodontics and Pediatric Dentistry School of Dentistry, University of Rio de Janeiro, Rio de Janeiro, RJ, Brazil

${ }^{5}$ Department of Orthodontics and Pediatric Dentistry School of Dentistry, University of Michigan, Ann Arbor, Michigan

${ }^{6}$ Department of Experimental and Clinical Biomedical Sciences, Radiodiagnostic Unit $\mathrm{n}$. 2, University of Florence, Florence, Italy

${ }^{7}$ Department of Orthodontics and Pediatric Dentistry, University of Michigan, Ann Arbor, Michigan

${ }^{8}$ School of Medicine, University of Michigan, Ann Arbor, Michigan

${ }^{9}$ Center for Human Growth and Development, University of Michigan, Ann Arbor, Michigan

\section{Correspondence}

Lorenzo Franchi, Department of Surgery and Translational Medicine, Section of Dentistry, Università degli Studi di Firenze, Firenze, Italy.

Email: Iorenzo.franchi@unifi.it

\section{Structured Abstract}

Objectives: To compare the three-dimensional maxillary dentoskeletal effects of a modified alternate rapid maxillary expansion and constriction facemask protocol (AltRAMEC/FM) with the traditional rapid maxillary expansion facemask protocol (RME/FM) performed in deciduous or early mixed dentition Class III patients.

Setting and sample population: Orthodontic Clinic of the Section of Dentistry of the Department of Surgery and Translational Medicine of the University of Florence. Thirty-four Class III patients were enrolled and allocated by alternating assignment to either Alt-RAMEC/FM or RME/FM therapy.

Materials and Methods: Prior to treatment, all patients were evaluated, and a cone beam computed tomography (CBCT) scan was acquired. After completion of the orthopaedic therapy (average interval 1.2 years), a follow-up CBCT scan was obtained. Anatomic landmark identification on the CBCTs and subsequent quantification of the changes were performed. The primary outcome variable was the anteroposterior displacement of the anterior nasal spine (ANS AP). The treatment groups were compared with independent samples $t$ tests.

Results: The patients in the two treatment groups showed a similar degree of compliance. No statistically significant differences were recorded for any variable when comparing the Alt-RAMEC/FM and RME/FM groups. In particular, the betweengroup difference for ANS AP was $0.0 \mathrm{~mm}(95 \% \mathrm{Cl}:-0.6 ; 0.7, \mathrm{P}=0.933)$.

Conclusions: Both Alt-RAMEC/FM and RME/FM produced favourable orthopaedic changes in Class III growing patients. Neither protocol was superior to the other in terms of maxillary protraction effectiveness.

\section{KEYWORDS}

3D imaging, class III malocclusion, dentofacial orthopedics

\section{1 | INTRODUCTION}

Class III malocclusion makes up just $7 \%$ of malocclusions worldwide, and the prevalence varies widely across geographic regions and racial/ethnic background. ${ }^{1}$ Despite this relatively low prevalence,
Class III disharmony is one of the most difficult and unpredictable malocclusions to treat in orthodontics.

Today the most common treatment protocol for correction of Class III malocclusion in growing patients is rapid maxillary expansion combined with facemask therapy (RME/FM). ${ }^{2}$ Despite the 
purported benefits of RME, the results of a recent systematic review showed that the expansion of the palate transversely does not per se improve maxillary protraction. ${ }^{3}$ The clinical effects of the facemask, which improve the Class III profile and malocclusion, can be attributed to the protraction of the maxilla as well as the downward and backward rotation of the mandible. ${ }^{4-6}$

In an effort to increase maxillary protraction and improve treatment outcomes in Class III patients, Erik Liou proposed a new orthopaedic treatment approach that combines alternating rapid maxillary expansion and constriction (Alt-RAMEC) with intraoral maxillary protraction springs. ${ }^{7,8}$

With the promising Alt-RAMEC results reported by Liou, ${ }^{7,8}$ additional investigators began testing this novel concept in combination with the extraoral facemask. In 2009, Do-deLatour et al ${ }^{9}$ found no significant difference in maxillary protraction when comparing the activation-deactivation/FM group and the traditional RME/FM group. On the other hand, in 2010 Isci et al ${ }^{10}$ reported nearly twice as much maxillary protraction for the Alt-RAMEC/FM protocol vs the RME/FM protocol. It should be noted, however, that the activation rate and duration of treatment were not consistent between these two studies.

Masucci et al ${ }^{11}$ in 2014 compared the RME/FM standard treatment approach with a modified 4-week Alt-RAMEC/FM protocol performed in deciduous dentition patients to maximize protraction orthopaedic effects. The investigators reported that both the Alt-RAMEC/FM and RME/FM groups showed favourable maxillary advancement effects compared to the control group, but the AltRAMEC/FM group demonstrated a significantly greater change $\left(\mathrm{SNA}+1.2^{\circ}\right)$ in sagittal maxillary position and intermaxillary relationships compared to the RME/FM group.

A randomized controlled trial (RCT) published by Liu and coworkers in $2015^{12}$ showed that a 7-week Alt-RAMEC/FM protocol increased the maxillary forward movement significantly with respect to the RME/FM protocol (A to Vertical Axis through Sella $+0.9 \mathrm{~mm}$ ).

The effectiveness of the Alt-RAMEC protocol both in association with facemask or other protraction methods was confirmed by a recent systematic review, ${ }^{13}$ although the authors emphasized the need for further studies to assess the stability of this treatment approach.

It should be noted, however, that only conventional 2D cephalometric studies evaluating the effects of the Alt-RAMEC/FM protocol vs the effects of the RME/FM are available in literature. Thus, the objective of the current study was to compare the three-dimensional maxillary dentoskeletal effects of a modified alternate rapid maxillary expansion and constriction facemask protocol (Alt-RAMEC/FM) with the traditional rapid maxillary expansion facemask protocol (RME/ FM) performed in deciduous or early mixed dentition Class III patients.

\section{2 | SUBJECTS AND METHODS}

Ethical approval for this prospective controlled study was obtained from the Pediatric Ethics Committee of the Region of Tuscany,
(\#2014/0000554). Informed consent was obtained from each patient's family prior to treatment.

A parallel quasi-randomized design with a 1:1 allocation ratio was implemented by alternating assignment of the Class III patients to either the Alt-RAMEC/FM protocol or the RME/FM protocol.

The inclusion criteria were as follows:

1. European ancestry (White/Caucasian).

2. Family history of Class III malocclusion.

3. Anterior crossbite or edge-to-edge incisor relationship.

4. Accentuated mesial step relationships of the primary second molars or Class III relationships of the permanent first molars.

5. Absence of CO-CR discrepancy (eg, pseudo-Class III malocclusions were excluded).

6. Deciduous or early mixed dentition.

7. Prepubertal skeletal maturation CS1 to $\mathrm{CS} 2^{14,15}$.

8. Absence of craniofacial anomalies (eg, cleft lip and/or palate).

Failure to meet the inclusion criteria resulted in exclusion from the study. Patients who met the inclusion criteria were enrolled in the study following a discussion and documentation of informed consent with the family.

Patient recruitment was conducted in the Orthodontic Clinic of the Section of Dentistry of the Department of Surgery and Translational Medicine of the University of Florence, from September 2011 to July 2016.

All patients received an expander and a facemask of consistent designs, as have been described in a previous study. ${ }^{11} \mathrm{~A}$ maxillary acrylic splint expander with soldered facemask hooks and a $0.2 \mathrm{~mm}$ per turn expansion screw (Leone A2620, Leone Orthodontic Products, Sesto Fiorentino, Firenze, Italy) was fabricated for each patient and bonded to the deciduous canines and deciduous molars. Parents were instructed about how to turn the expander.

Following the expansion phase, a petit-design facemask (Dynamic facemask, Leone Orthodontic Products, Sesto Fiorentino, Firenze, Italy) was delivered to each patient to initiate the maxillary protraction phase of the treatment. Elastics were attached to the facemask bilaterally, each delivering 400-500 g of orthopaedic force in a downward and forward direction of about $30^{\circ}$ to the occlusal plane. $^{16}$

The Alt-RAMEC/FM protocol consisted of:

1. Delivery of maxillary acrylic splint expander with soldered facemask hooks;

2. Activation turns ( $2 \times$ per day, corresponding to $0.4 \mathrm{~mm}$ of expansion) for maxillary expansion for 1 week;

3. Deactivation turns ( $2 \times$ per day) for maxillary constriction for 1 week;

4. Repeat steps 2 and 3 so that the Alt-RAMEC cycle occurred three times and lasted for a total of 6 weeks;

5. Activation turns ( $2 \times$ per day) until individualized transverse dimension was achieved; 
6. Delivery of facemask and elastics and instruction of patients to wear the facemask for 14 hours per day for 6 months, then at night only for additional 6 months;

7. Patients were treated to achieve a positive dental overjet, with the goal of overcorrecting to a Class II molar relationship.

The RME/FM protocol consisted of:

1. Delivery of maxillary acrylic splint expander with soldered facemask hooks;

2. Activation turns ( 1 or $2 \times$ per day, corresponding to 0.2 or $0.4 \mathrm{~mm}$ of expansion, respectively) until individualized transverse dimension was achieved;

3. Delivery of facemask and elastics and instruction of patients to wear the facemask for 14 hours per day for 6 months, then at night only for an additional 6 months;

4. Patients were treated to achieve a positive dental overjet, with the goal of overcorrecting to a Class II molar relationship.

A three-dimensional CBCT image was acquired at 2 time points for each patient. The first CBCT was taken before treatment (T1) and a post-treatment $\mathrm{CBCT}$ (T2) was acquired 1 to 6 months after completion of active treatment. The CBCTs were taken exclusively for patients involved in this study.

The 3D analysis was performed using the open source software ITK-SNAP (version 2.2, www.itksnap.org) and 3D Slicer (version 3.1, www.slicer.org). The original DICOM files were converted to de-identified GIPL files using ITK-SNAP. The CBCTs were acquired through 2 radiology centres, so the files were converted and standardized to an isotropic voxel size of $0.5 \times 0.5 \times 0.5 \mathrm{~mm}$, using 3DSlicer (downsize image tool in Slicer). Subsequently, 3D image analysis was performed through the following steps (Figure S1):

1. Construction of $3 D$ volumetric label maps (segmentations) and $3 D$ surface models of the T1 scan: Using a combination of the 3D Slicer extension Intensity Segmenter and ITK-SNAP, 3D segmentations of the GIPL files were constructed. The automatic segmentation was generated in 3D Slicer applying a range file that describes the different ranges of density with corresponding colours to be used for the software to build the segmentation. The ITK-SNAP software allows the user to adjust the parameters of the automated features and to edit contours of the segmentation directly. In ITK-SNAP, the segmentation also was cropped and cleaned. Then, using the extension model maker in 3D Slicer, the T1 segmentations were converted to $3 \mathrm{D}$ surface models (vtk files).

2. Head orientation in 3D Slicer: $3 D$ Slicer provides a fixed 3D coordinate system with three orthogonal planes denoted by yellow, red and green colours; these planes were used as a reference to orient the T1 models of each patient. The Transforms tool was used to orient the T1 model based on the following landmarks and planes. Glabella, Crista Galli and Basion were used to define the midsagittal plane and the axial plane was defined by the bilateral structures of Orbitale and Porion (Frankfort horizontal plane).

Using the axial, coronal and sagittal views of the model, the T1 model was rotated and translated until the midsagittal plane coincided with the yellow plane and the axial plane with the red plane. ${ }^{17}$ Once oriented properly, the matrix generated from this transformation was saved and applied to the scan and segmentation for T1, providing the same head orientation to them. This process was repeated for each patient, thus achieving a common head orientation within the 3D Slicer coordinate system for all patients.

3. Manual approximation of T1 and T2: The GIPL files for T1 and T2 were loaded into 3D Slicer. Using the Transform tool in 3DSlicer, the T1 oriented GIPL was selected as the stable file. The T2 file was moved (translated and rotated) in the left-right, posterior-anterior and inferior-superior planes of space to best approximate the superimposition of the T1 and T2 GIPL files on the cranial base (used as reference to achieve the superimposition).

4. Construction of $3 D$ volumetric label maps (segmentations) of the approximated T2 scan: The same procedure described in the step \#1 was used to construct T2 segmentations. Two segmentations were generated for the approximated T2 scan: the full head and the cranial base segmentations. The cranial base segmentation was used in the next step to indicate to the software which regions it should look for corresponding voxels in T1 and T2 to perform the superimposition.

5. Voxel-based registration of GIPL files using the cranial base as reference: The oriented T1 and the T2 approximated GIPL files with their corresponding segmentations were loaded into 3DSlicer. The 3D voxel-based registration (CMF registration tool) was used to align the T1 and T2 GIPL files automatically by utilizing corresponding voxels in the cranial base to achieve a reliable and reproducible superimposition of the 2 time points. Once this automated voxel-based registration was completed, the registered files (scan and segmentation) were used for the subsequent steps.

6. Placement of pre-labelled landmarks on the segmentations: The segmentations were cleaned, and the mandible was removed from the segmentation to allow better visualization of the maxilla and maxillary dentition. The following eight landmarks were identified: 1 and 2) Orbitale right and left ( $\mathrm{Or}_{R}$, Or $\mathrm{L}_{\mathrm{L}}$ ), most inferior point of the orbital concavity in a frontal view, centred anterior-posteriorly on the orbital rim from the superior view; 3 and 4) Infrazygomatic arch right and left $\left(\mathrm{IZ}_{\mathrm{R}}, \mathrm{IZ} \mathrm{Z}_{\mathrm{L}}\right)$, greatest point of convexity where the horizontal and sagittal components of the zygomatic arch intersect in an inferior view; 5 and 6) Incisal tip of primary canine right and left $\left(C_{R}, C_{L}\right)$; 7) Posterior nasal spine (PNS); 8) Anterior nasal spine (ANS). Attention to detail was taken to clean these regions throughout the segmentation process.

The segmentation of the cranial base and maxilla was performed in a single colour (green). The eight landmarks for T1 and T2 were 
pre-labelled in a different colour (red) to facilitate data computation in future steps and minimize landmark placement error (prelabelling). ${ }^{18}$ Sagittal, axial and coronal slices of the greyscale image as well as the $3 \mathrm{D}$ reconstruction of the image were used for landmark positioning in the ITK-SNAP software.

7. Generation of 3D Models (vtk files) in 3DSlicer: Using the Model Maker tool in 3DSlicer, 3D surface models (Figure 1) were generated for the segmented head and pre-labelled landmarks for both T1 and T2 for each patient.

8. Landmark based quantitative assessments in Q3DC: The Q3DC tool was used to calculate the millimetric changes from T1 to T2. Landmarks were placed on the 3D surface models, and the displacement of the following landmarks was reported in anteriorposterior and superior-inferior changes as well as 3D distances (midpoints were generated for bilateral landmarks): Orbitale Midpoint $\left(\mathrm{Or}_{M}\right)$, Infrazygomatic Midpoint $\left(\mathrm{I}_{\mathrm{M}}\right)$, Canine Midpoint $\left(C_{M}\right)$, and Anterior Nasal Spine (ANS). The changes on the palatal plane were measured by calculating the pitch angle between the palatal plane (ANS-PNS) at T1 and at T2. The anteroposterior displacement of anterior nasal spine (ANS AP) was selected as primary outcome variable while all other variables were considered as secondary outcome variables.

Q3DC tool also was used to obtain the starting form measurements for all T1 models: Vertical, Nasion (N)-Palatal Plane (ANSPNS); Sagittal, ANS-Nasion Perpendicular (NPerp); Transverse, Intercanine Width (CC 3D); and Maxillary Length (ANS-PNS 3D).

9. Generation of semitransparent overlays and colormaps for visualization: Semitransparent overlays of the T1 and T2 models were

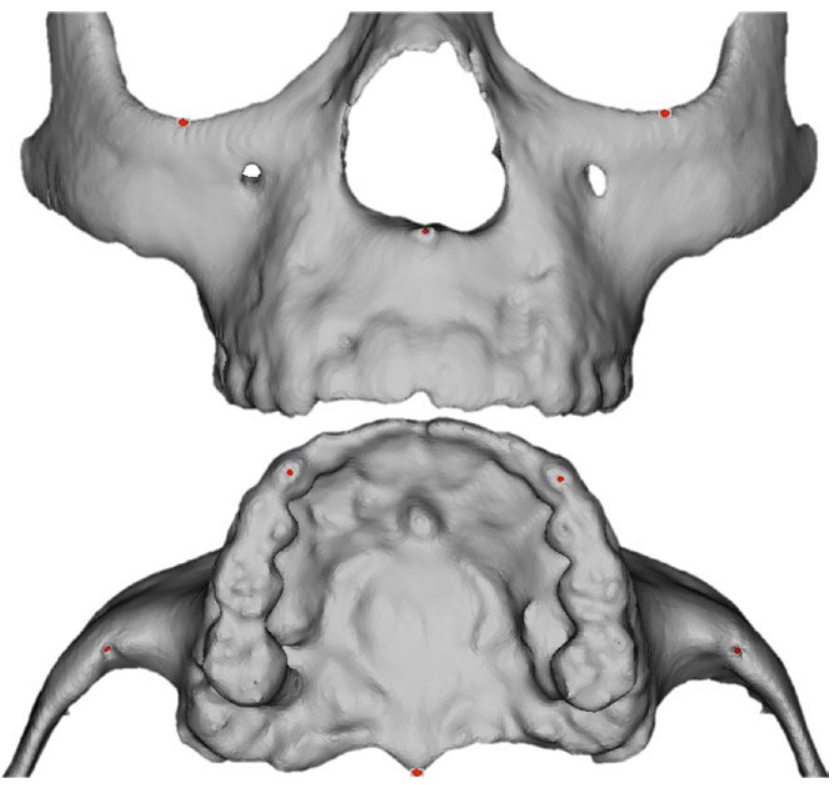

FIGURE 1 Surface models generated in 3DSlicer. A, Pre-labelled maxilla model, frontal view; $B$, Pre-labelled maxilla model, inferior view [Colour figure can be viewed at wileyonlinelibrary.com] created for visualization. Additionally, using the Model-to-Model Distance and Shape Population Viewer, colormaps were generated to visualize the changes from T1 to T2.

One examiner (BHF) performed steps 1 through 5 while a second examiner (ACR) checked steps 1 through 5 and performed steps 6 through 9. Both examiners were blinded to the treatment assignment.

Removable appliances in orthodontics, such as the facemask, require good compliance to achieve successful treatment outcomes. As anticipated, the degree of compliance varied among patients in this study. To monitor the patients throughout treatment, the clinician recorded compliance rate at each appointment, which occurred every 5 weeks. Parents were asked how often and how long the facemask was worn each day and it was assessed as moderate or excellent. Excellent compliance was noted if the patient wore the facemask a total of 14 hours throughout the day and night during the first 6 months, followed by 6 months of nighttime wear. If excellent compliance was not achieved, the patient's compliance was classified as moderate.

The sample size was computed considering $\alpha=0.05$, power $=0.80$, an effect size of 1 calculated from the standard deviation of $1.6^{\circ}$ for the variable S-N-A derived from the data of the study by Masucci et al ${ }^{11} \mathrm{~A}$ sample size of 40 patients (20 patients for each group) was determined to be adequate, allowing for a $15 \%$ dropout rate.

Patient allocation was performed by alternating assignment to either one of the two treatment protocols. The two examiners who carried out the $3 \mathrm{D}$ analysis and the statistician were blinded as to treatment group assignment.

\section{1 | Statistical analysis}

Intraclass correlation coefficient (ICC) two-way mixed with absolute agreement was used to test the intra-rater agreement of the examiner (ACR) on 15 patients. The variables were re-measured after 1 week. Random error was evaluated using Springate's method of moments estimator (MME). ${ }^{19}$

Mean and standard deviation were used for quantitative variables, while frequency and percentage were calculated for qualitative variables.

To test differences at baseline between the two groups, independent samples $t$ tests were used for the quantitative variables and Fisher's exact tests for dichotomous variables.

To test differences between the two groups on the outcome variables, independent samples $t$ tests were applied reporting $P$ values, differences between treatments and $95 \%$ confidence intervals of the differences. For the primary outcome variable ANS AP (ie, the anteroposterior displacement of anterior nasal spine), equality of variance was assessed using Levene's test, and an analysis of the residuals was performed to assess their normality using the Shapiro-Wilk test. If at least one of the previous tests was significant, a sensitivity analysis was performed using the Mann-Whitney $\mathrm{U}$ test. 


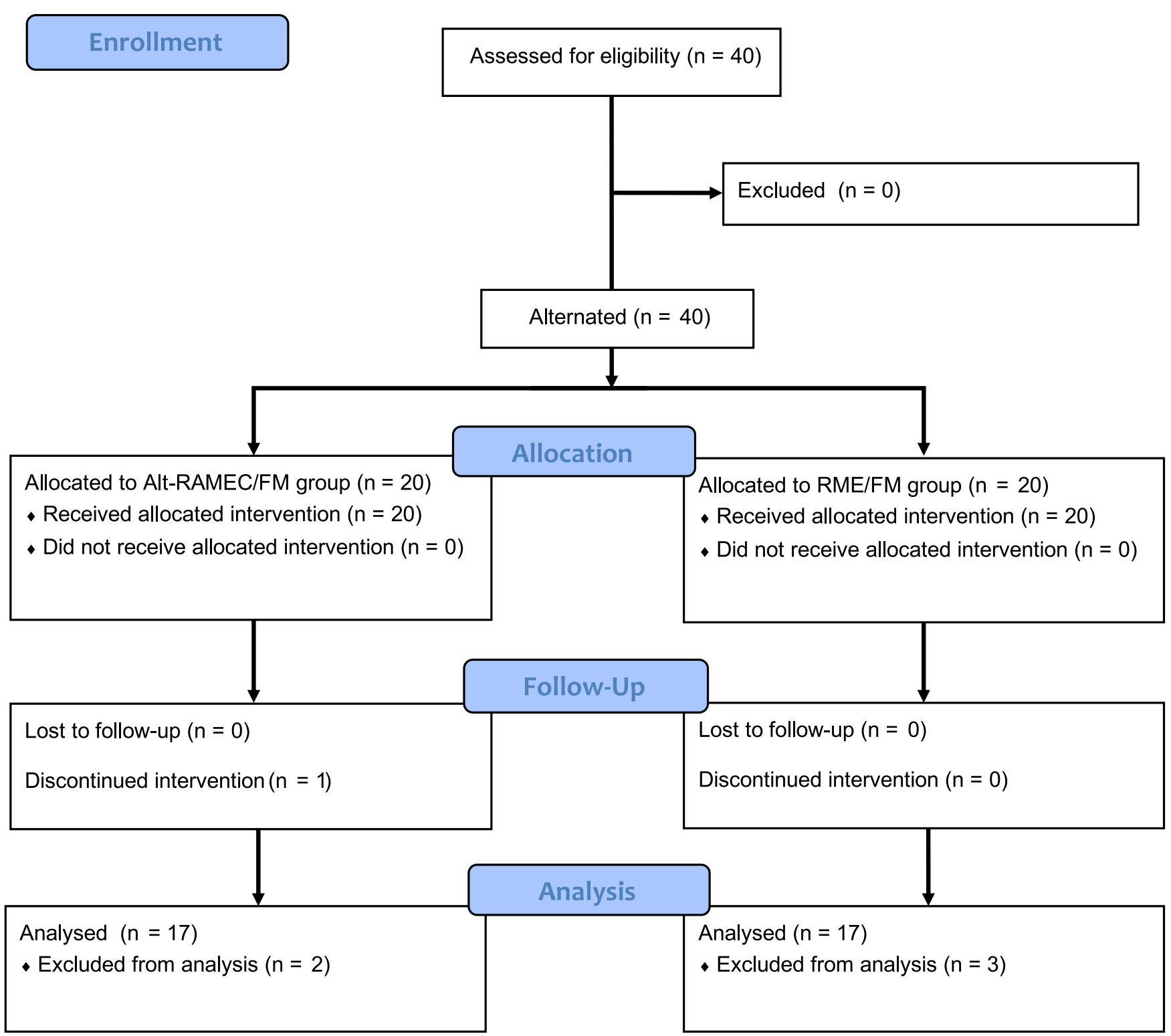

FIGURE 2 CONSORT flow diagram [Colour figure can be viewed at wileyonlinelibrary.com]

For variable ANS AP, a linear model using treatment group, gender, compliance and age as predictor variables also was applied.

JMP® version 13.0.0 2016 (SAS Institute Inc., Cary, NC, USA) and MedCalc Statistical Software version 12.7.8 (MedCalc Software bvba, Ostend, Belgium) were used for the statistical computations.

\section{3 | RESULTS}

Initially, 40 patients were enrolled in the study (Figure 2). The baseline characteristics of both groups are reported in Table 1. The Alt-RAMEC/FM group consisted of 20 patients (11 females, 9 males). The average pre-treatment $\mathrm{CBCT}$ (T1) acquisition age was $6.0 \pm 0.9$ years. The RME/FM group was comprised of 20 patients (12 females, 8 males). The average pre-treatment CBCT (T1) acquisition age was $6.3 \pm 0.8$ years. No statistically significant differences between the two groups were found for the 4 starting form measurements.

One patient of the Alt-RAMEC/FM discontinued intervention after 3 months of therapy due to excessive gingival inflammation. Five patients were excluded from analysis. Reasons for exclusion were the following: In the Alt-RAMEC/FM Group 2, patients' parents refused to take a second CBCT. In the RME/FM group 2, patients' parents refused to take a second $C B C T$ while in one patient the second CBCT presented with movement artefact, rendering the scan inadequate for landmark identification. Patient recruitment was conducted in the Orthodontic Clinic of the Section of Dentistry of the Department of Surgery and Translational Medicine of the University of Florence from September 2011 to July 2016.

As a result, 34 patients (18 females, 16 males) remained with 17 patients in each treatment group. In the Alt-RAMEC/FM group, the average post-treatment $\mathrm{CBCT}$ (T2) age was $7.5 \pm 1.0$ years, and the 
TAB LE 1 Statistical comparisons for gender, age for CBCT at T1, and 4 variables at baseline (T1)

\begin{tabular}{|c|c|c|c|}
\hline Variable & $\begin{array}{l}\text { Alt-RAMEC/FM } \\
\mathrm{N}=20\end{array}$ & $\begin{array}{l}\text { RME/FM } \\
\mathrm{N}=20\end{array}$ & $P$-value \\
\hline $\begin{array}{l}\text { Gender } \\
\text { (Female) }\end{array}$ & 11 (55\%) & $12(60 \%)$ & 1.000 \\
\hline $\begin{array}{l}\text { Age CBCT at } \\
\text { T1 (y) }\end{array}$ & $6.0(0.9)$ & $6.3(0.8)$ & 0.250 \\
\hline N PalPI SI (mm) & $41.4(2.2)$ & $41.8(2.3)$ & 0.522 \\
\hline $\begin{array}{l}\text { ANS-NPerp AP } \\
(\mathrm{mm})\end{array}$ & $0.4(2.6)$ & $1.0(2.3)$ & 0.386 \\
\hline CC 3D (mm) & $28.3(1.7)$ & $28.1(2.7)$ & 0.744 \\
\hline $\begin{array}{l}\text { ANS-PNS 3D } \\
(\mathrm{mm})\end{array}$ & $42.4(2.0)$ & $42.8(2.8)$ & 0.647 \\
\hline
\end{tabular}

$\mathrm{AP}$, antero-posterior; $\mathrm{CBCT}$, cone beam computed tomography; $\mathrm{CC}$, intercanine width; NPerp, nasion perpendicular; PalPI, palatal plane; SI, supero-inferior.

average T1-T2 interval was $1.6 \pm 0.2$ years. Treatment duration in this group was $1.2 \pm 0.1$ years. In the RME/FM group, the average post-treatment $\mathrm{CBCT}(\mathrm{T} 2)$ age was $8.0 \pm 0.8$ years, and the average T1-T2 interval was $1.5 \pm 0.3$ years. Treatment duration in this group was $1.1 \pm 0.2$ years.

The patients of the two treatment groups showed a similar degree of compliance. In the Alt-RAMEC/FM group, 9 patients (47\%) showed excellent compliance, and 10 patients (53\%) exhibited moderate compliance. In the RME/FM group, 9 patients (45\%) showed excellent compliance, 11 patients (55\%) exhibited moderate compliance. No statistically significant differences were found between the two groups in terms of degree of compliance $(P=1.0)$.

The ICCs are reported in Table 2. The values varied from 0.84 to 0.98 , indicating excellent intra-rater agreement. ${ }^{20}$ The MME random error measurements ranged from 0.12 to $0.32 \mathrm{~mm}$. Inter-observer agreement, using similar methods and involving the same examiner (ACR), had been tested already in a previous study ${ }^{17}$ that revealed excellent agreement.

Statistical comparisons for the T1-T2 changes of the Alt-RAMEC/ FM and RME/FM groups are reported in Table 3. There were no statistically significant differences between the two treatment groups. Levene's test and the Shapiro-Wilk test were not significant for the primary outcome variable ANS AP.

The amount of anterior-posterior and superior-inferior displacement of the maxilla was proportional at about 1:1 ratio in both groups. The maxilla, as measured at ANS, was protracted by $2.0 \mathrm{~mm}$ in both groups and was displaced inferiorly by $2.4 \mathrm{~mm}$ in the AltRAMEC/FM group and $2.1 \mathrm{~mm}$ in the RME/FM group. Less skeletal displacement was observed at the infrazygomatic midpoint, with the Alt-RAMEC/FM group showing $1.0 \mathrm{~mm}$ anterior and $2.0 \mathrm{~mm}$ inferior displacement, and the RME/FM group showing $1.2 \mathrm{~mm}$ anterior and $1.7 \mathrm{~mm}$ inferior displacement. The greatest changes were found at the primary canines with a forward displacement of $3.4 \mathrm{~mm}$ and an inferior displacement of $4.2 \mathrm{~mm}$ in the Alt-RAMEC/FM group and a forward displacement of $3.5 \mathrm{~mm}$ and an inferior displacement of
TAB LE 2 Mean differences between the two measures, intraclass correlation coefficient (ICC) with $95 \%$ confidence interval $\mathrm{Cl}$ ) and random error evaluated using the method of moments estimator (MME)

\begin{tabular}{|c|c|c|c|c|}
\hline Variable & Mean diff. & ICC & $95 \% \mathrm{Cl}$ & $\begin{array}{l}\text { MME (mm } \\
\text { or degrees) }\end{array}$ \\
\hline $\mathrm{Or}_{\mathrm{M}} \mathrm{AP}(\mathrm{mm})$ & 0.15 & 0.87 & $0.65 ; 0.95$ & 0.25 \\
\hline $\mathrm{Or}_{\mathrm{M}} \mathrm{SI}(\mathrm{mm})$ & 0.19 & 0.84 & $0.58 ; 0.94$ & 0.32 \\
\hline $\mathrm{Or}_{\mathrm{M}} 3 \mathrm{D}(\mathrm{mm})$ & -0.02 & 0.84 & $0.57 ; 0.94$ & 0.29 \\
\hline $\mathrm{IZ}_{\mathrm{M}} \mathrm{AP}(\mathrm{mm})$ & 0.10 & 0.89 & $0.70 ; 0.96$ & 0.25 \\
\hline $\mathrm{IZ}_{\mathrm{M}} \mathrm{SI}(\mathrm{mm})$ & 0.10 & 0.87 & $0.67 ; 0.96$ & 0.28 \\
\hline $\mathrm{IZ}_{\mathrm{M}} 3 \mathrm{D}(\mathrm{mm})$ & -0.06 & 0.88 & $0.67 ; 0.96$ & 0.27 \\
\hline$C_{M} A P(m m)$ & 0.22 & 0.98 & $0.67 ; 0.99$ & 0.13 \\
\hline $\mathrm{C}_{\mathrm{M}} \mathrm{SI}(\mathrm{mm})$ & 0.14 & 0.98 & $0.93 ; 0.99$ & 0.28 \\
\hline$C_{M} 3 D(m m)$ & 0.03 & 0.98 & $0.95 ; 0.99$ & 0.20 \\
\hline ANS AP (mm) & 0.06 & 0.98 & $0.94 ; 0.99$ & 0.12 \\
\hline ANS SI (mm) & 0.10 & 0.95 & $0.87 ; 0.98$ & 0.22 \\
\hline ANS 3D (mm) & -0.04 & 0.97 & $0.91 ; 0.99$ & 0.20 \\
\hline $\begin{array}{l}\text { ANS-PNS } \\
\text { Angle }\left({ }^{\circ}\right)\end{array}$ & 0.09 & 0.98 & $0.95 ; 0.99$ & 0.19 \\
\hline
\end{tabular}

$\mathrm{AP}$, antero-posterior; diff., difference; SI, supero-inferior.

$3.9 \mathrm{~mm}$ in the RME/FM group. As illustrated in Figure 3, patients in both groups varied in the amount and direction of dentoskeletal change as a response to treatment.

As for the results of the linear regression analysis, no significant predictor was identified for the primary outcome variable ANS AP. Adjusted difference for ANS AP between the 2 treatments was $0.00 \mathrm{~mm}$ (95\%Cl: $-0.73 ; 0.73)$.

\section{4 | DISCUSSION}

The present study evaluated the three-dimensional maxillary dentoskeletal effects of a 6-week Alt-RAMEC/FM protocol with respect to the conventional RME/FM protocol in Class III growing patients. To our knowledge, no previous study analyzed the three-dimensional maxillary changes produced by the Alt-RAMEC/FM protocol vs the RME/FM protocol.

In this study, we choose to treat Class III patients during the early developmental stage, according to the study by Masucci et $\mathrm{al}^{11,}$ for two reasons. First, the forces generated during the repetitive weekly expansion/constriction protocol could produce negative periodontal effects and increase the risk of root resorption on maxillary first premolars and permanent molars ${ }^{13}$. These side effects could be avoided if the expansion/constriction forces were applied on the deciduous teeth. Secondly, the deciduous dentition phase coincides with a stage of skeletal development that is considered to be optimal for the correction of dentoskeletal Class III malocclusion as a good response to maxillary protraction is achieved during the prepubertal stage of skeletal maturation. ${ }^{14}$ 


\begin{tabular}{|c|c|c|c|c|c|}
\hline Variable & $\begin{array}{l}\text { Alt-RAMEC/FM } \\
\mathrm{N}=17\end{array}$ & $\begin{array}{l}\text { RME/FM } \\
N=17\end{array}$ & Differences & $95 \% \mathrm{Cl}$ & $P$-value \\
\hline $\operatorname{Or}_{M} A P(m m)$ & $0.8(0.8)$ & $1.3(0.9)$ & -0.5 & $-1.1 ; 0.1$ & 0.095 \\
\hline $\mathrm{Or}_{\mathrm{M}} \mathrm{SI}(\mathrm{mm})$ & $-1.0(0.6)$ & $-0.6(0.6)$ & -0.3 & $-0.8 ; 0.1$ & 0.127 \\
\hline $\operatorname{Or}_{M} 3 \mathrm{D}(\mathrm{mm})$ & $1.6(0.5)$ & $1.7(0.7)$ & -0.1 & $-0.5 ; 0.3$ & 0.634 \\
\hline$I Z_{M} A P(m m)$ & $1.0(0.7)$ & $1.2(0.8)$ & -0.2 & $-0.7 ; 0.3$ & 0.522 \\
\hline $\mathrm{IZ}_{\mathrm{M}} \mathrm{SI}(\mathrm{mm})$ & $-2.0(0.9)$ & $-1.7(0.8)$ & -0.3 & $-0.9 ; 0.3$ & 0.293 \\
\hline $\mathrm{IZ}_{\mathrm{M}} 3 \mathrm{D}(\mathrm{mm})$ & $2.5(0.6)$ & $2.2(0.7)$ & 0.3 & $-0.2 ; 0.7$ & 0.299 \\
\hline$C_{M} A P(m m)$ & $3.4(1.2)$ & $3.5(1.2)$ & -0.1 & $-1.0 ; 0.7$ & 0.794 \\
\hline $\mathrm{C}_{\mathrm{M}} \mathrm{SI}(\mathrm{mm})$ & $-4.2(1.3)$ & $-3.9(1.8)$ & -0.3 & $-1.3 ; 0.9$ & 0.642 \\
\hline$C_{M} 3 D(m m)$ & $5.6(0.9)$ & $5.5(1.6)$ & 0.1 & $-0.8 ; 1.0$ & 0.781 \\
\hline ANS AP (mm) & $2.0(1.0)$ & $2.0(0.9)$ & 0.0 & $-0.7 ; 0.6$ & 0.933 \\
\hline ANS SI (mm) & $-2.4(1.0)$ & $-2.1(1.2)$ & -0.3 & $-1.1 ; 0.4$ & 0.403 \\
\hline ANS 3D (mm) & $3.4(0.7)$ & $3.2(1.0)$ & 0.2 & $-0.3 ; 0.8$ & 0.421 \\
\hline $\begin{array}{l}\text { ANS-PNS } \\
\text { Angle }\left({ }^{\circ}\right)\end{array}$ & $-0.2(1.5)$ & $0.2(1.5)$ & -0.5 & $-1.5 ; 0.6$ & 0.358 \\
\hline
\end{tabular}

TABLE 3 Statistical comparisons for the T1-T2 changes

$\mathrm{AP}$, antero-posterior; SI, supero-inferior.

Using a newly developed extension in 3D Slicer, ${ }^{21}$ the Q3DC tool was used for landmark identifications in T1 and T2 for each patient. The Q3DC extension then calculated the three-dimensional change at each landmark and decomposed the measurement into component parts, providing the magnitude (millimetric) and direction of change. In contrast to the quantitative assessments using Q3DC, the semitransparent overlays in Figure 3 provided a qualitative visual representation of treatment results, respectively for the AltRAMEC/FM and RME/FM groups.

The individual variability of patients' response to therapy also is noteworthy, indicating other possible underlying factors related to treatment response rather than to the therapy of choice. While both treatment protocols led to marked maxillary protraction in some patients, the vertical growth component in other patients treated with the same protocol was obvious.

When comparing descriptive statistics across the two groups in the present study, the Alt-RAMEC/FM group and RME/FM group had similar demographics and starting form. When evaluating treatment outcomes, no significant difference was detected between the Alt-RAMEC/FM and RME/FM groups. Measured at ANS, the 3D displacement of the maxilla with growth and response to treatment was on average $3.4 \mathrm{~mm}$ in the Alt-RAMEC/FM group and $3.2 \mathrm{~mm}$ in the RME/FM group. In both groups, the maxillary changes measured at ANS occurred on average $2.0 \mathrm{~mm}$ in the anterior direction $(2.0 \pm 1.0 \mathrm{~mm}$ in the Alt-RAMEC/FM group and $2.0 \pm 0.9 \mathrm{~mm}$ in the RME/FM group). A similar amount of maxillary displacement of ANS occurred in a downward direction $(-2.4 \pm 1.0 \mathrm{~mm}$ in the Alt-RAMEC/ FM group and $-2.1 \pm 1.2 \mathrm{~mm}$ in the RME/FM group).

This finding is not consistent with the results reported by DodeLatour et al, ${ }^{9}$ who found no significant differences in maxillary advancement between the Alt-RAMEC/FM group $(1.8 \mathrm{~mm})$ and the RME/FM group (2.6 mm) in two 8.5-year-old samples of Class III patients. It is interesting to note that Do-deLatour et al $^{9}$ applied a more aggressive Alt-RAMEC protocol $(1 \mathrm{~mm} / \mathrm{d}$ for 7 weeks, similar to the original protocol proposed by $\left.\mathrm{Liou}^{7}\right)$ than that used in the current study $(0.4 \mathrm{~mm} / \mathrm{d}$ for 6 weeks).

Also, authors found more favourable treatment outcomes in patients undergoing alternating expansion/constriction protocols with respect to the conventional RME/FM protocol. ${ }^{10-12}$ In particular, Isci et al ${ }^{10}$ evaluated the effects of an Alt-RAMEC/FM treatment in 11-year-old Class III patients by applying an Alt-RAMEC protocol that was similar to the one used in the current study $0.4 \mathrm{~mm} / \mathrm{d}$ for 4 weeks). The authors reported more favourable maxillary advancement in the Alt-RAMEC/FM group (4.1 mm) compared to the RME/ FM group $(2.3 \mathrm{~mm})$. Similar results were published by Liu et al, ${ }^{12}$ who found an increased maxillary forward movement with the AltRAMEC/FM protocol ( $1 \mathrm{~mm} / \mathrm{d}$ for 7 weeks) with respect to the conventional RME/FM protocol (A to Vertical Axis through Sella 3.0 and $2.1 \mathrm{~mm}$, respectively) both performed in 10-year-old Class III patients.

The age of the patients at the start of treatment also could be a factor contributing to the difference in clinical outcomes between the present study and the studies by Isci et $\mathrm{al}^{10}$ and Liu et $\mathrm{al}^{12}$ Masucci et al, ${ }^{11}$ who evaluated the treatment outcomes of the AltRAMEC $(0.4 \mathrm{~mm} / \mathrm{d}$ for 6 weeks) vs RME protocols combined with FM in 6-year-old Class III patients, also reported data that is not consistent with the results of the present study. The investigators found that the Alt-RAMEC/FM group showed a significantly greater maxillary advancement (SNA $2.7^{\circ}$ ) with respect to the RME/FM group (SNA $\left.1.5^{\circ}\right)$.

When explaining these dissimilarities in terms of maxillary protraction, it should be stressed that there are at least 2 main differences in terms of methodology between the present study and the previous studies ${ }^{10-12}$ that analyzed the effects of the Alt-RAMEC/ FM protocol vs the RME/FM protocol. Firstly, the present study performed a three-dimensional evaluation of the effects of treatment. The T1-T2 changes were assessed after superimposition of the scan 

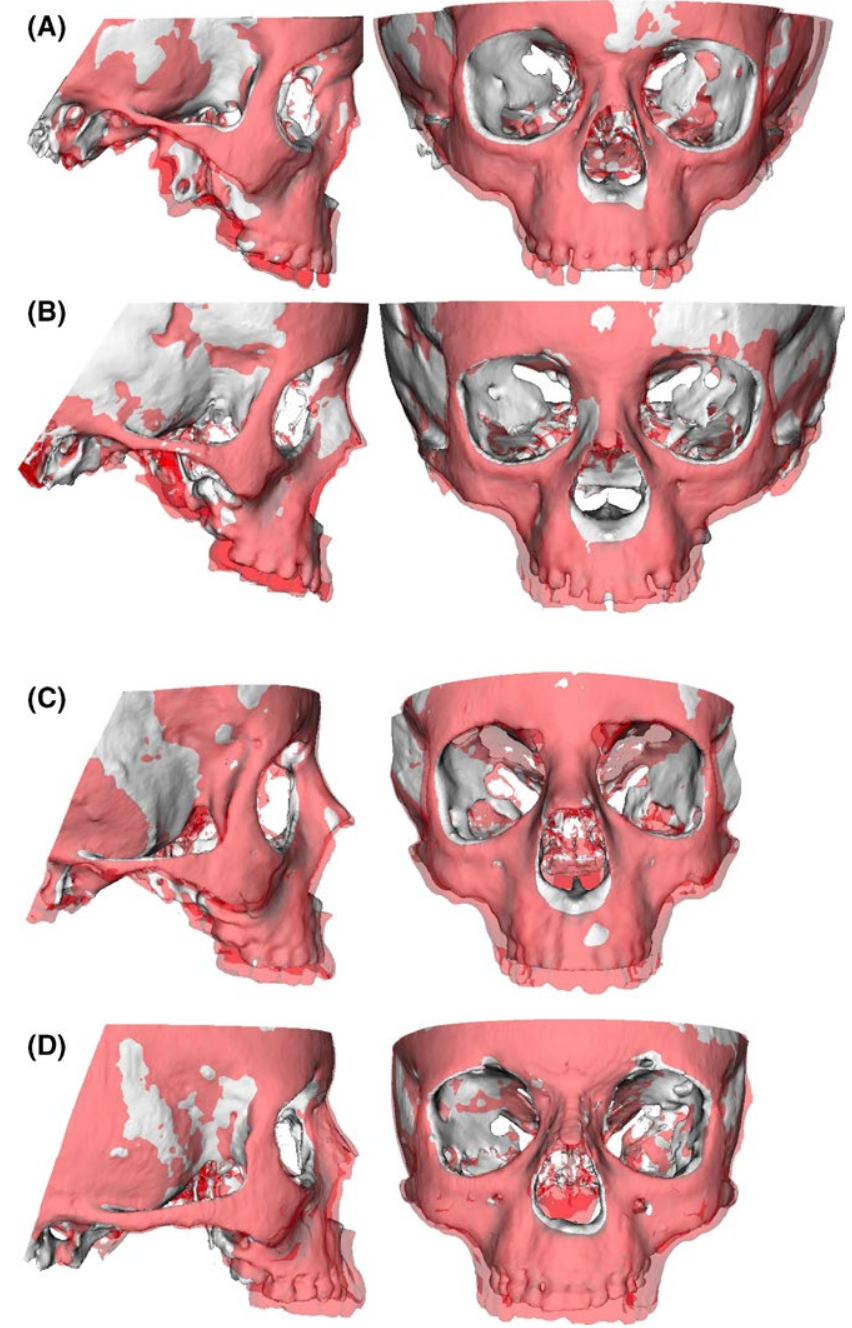

FIGURE 3 Individual variability in patient response to therapy with Alt-RAMEC, as shown by semitransparent overlays of 3D surface models superimposed in the cranial base. A, patient who presented predominantly vertical changes at the orbit and zygoma, as shown in close-up view of the floor of the orbit. The maxillary growth also was predominantly vertical. B, patient who presented favourable maxillary anterior protraction with some vertical displacement with growth. C, patient who presented predominantly vertical changes at the orbit and zygoma, as shown in close-up view of the floor of the orbit. The maxillary growth also was predominantly vertical. D, patient who presented favourable maxillary anterior protraction [Colour figure can be viewed at wileyonlinelibrary.com]

at T1 and the scan at T2 on the stable structures of the cranial base. This procedure allowed for more reliable and precise analysis results with respect to the previous $2 \mathrm{D}$ studies. ${ }^{10-12}$ Secondly, all these studies used point $A$ to evaluate maxillary advancement. It should be emphasized, however, that the reliability of the localization of point A can be influenced by the position of the upper central incisors, especially during the eruption period (Figure 4). For these reasons, the present study adopted the ANS point to assess maxillary movement.

If the amount of maxillary advancement reached with both protocols $(2.0 \mathrm{~mm})$ is considered, it is consistent with the data reported by several authors $\mathrm{s}^{4,5,16}$ in the literature on the effects of RME/FM treatment (1.5-3 mm) and it confirms the efficacy of FM protraction therapy produced by both Alt-RAMEC and RME protocols.

Although not as great in magnitude as the maxillary change, the zygomatic movement, as measured from the infrazygomatic landmarks, followed the same downward and forward trend (Figure 3). This study measured changes at the infrazygomatic midpoint to evaluate the zygomatic changes and to eliminate the expansion variable when determining the $3 \mathrm{D}$ distances from $\mathrm{T} 1$ to T2. The 3D displacement of infrazygomatic midpoint was on average $2.5 \pm 0.6 \mathrm{~mm}$ in the Alt-RAMEC/FM group and $2.2 \pm 0.7 \mathrm{~mm}$ in the RME/FM group. Different from the maxilla, the growth and treatment changes at the zygoma occurred more in an inferior direction $(-2.0 \pm 0.9$ and $-1.7 \pm 0.8 \mathrm{~mm}$, respectively in the Alt-RAMEC/FM and RME/FM groups) than in anterior direction $(1.0 \pm 0.7$ and $1.2 \pm 0.8 \mathrm{~mm}$, respectively in the Alt-RAMEC/FM and RME/FM groups).

The primary canines, as measured by the generated midpoint, moved in the Alt-RAMEC/FM group and in the RME/FM group $5.6 \mathrm{~mm}(3 \mathrm{D})$ and $5.5 \mathrm{~mm}(3 \mathrm{D})$ respectively and $3.4 \mathrm{~mm}(\mathrm{AP})$ and $3.5 \mathrm{~mm}$ (AP) of that movement was in the anterior direction. These data provide insight into the amount of anchorage loss that occurred despite the use of the bonded expander in the primary dentition. Knowing that the maxilla came forward $2.0 \mathrm{~mm}$ in both groups and the canines came forward $3.5 \mathrm{~mm}$, it can be postulated that there was $1.5 \mathrm{~mm}$ of forward dental movement, or anchorage loss, during protraction therapy. This mesial movement of the dentition can improve the Class III dental relationship, but it may contribute to anterior crowding or upper incisor flaring that is reported in the literature $^{3}$ as a common side effect of facemask therapy. It should be noted that the permanent maxillary first molar, if present, was not used for anchorage.

Another factor that may have led to no significant differences in treatment outcomes between the groups could be related to the timing of treatment. The Alt-RAMEC protocol ${ }^{7}$ originally was proposed to disarticulate the maxilla and produce more effective midfacial protraction. In the current study, however, both Alt-RAMEC and RME protocols were performed at an early age when the circummaxillary sutures are not interdigitated. ${ }^{22}$ In fact, the greatest amount of maxillary protraction has been reported in studies ${ }^{10,12}$ that started Alt-RAMEC treatment closer to puberty when the circummaxillary sutures have become heavily interdigitated. ${ }^{22}$ At that stage, the Alt-RAMEC protocol may be more effective in loosening the maxillary sutures than conventional RME.

The results of this study suggest that both Alt-RAMEC/FM and RME/FM protracted the maxilla in a forward and downward direction effectively; however, limitations of the current study were the absence of randomization and the lack of an untreated control group or FM-only group without expansion. Moreover, long-term follow-up of these patients would provide clinicians with valuable information to evaluate if the there is a difference in the amount of maxillary protraction that is retained following active growth. 


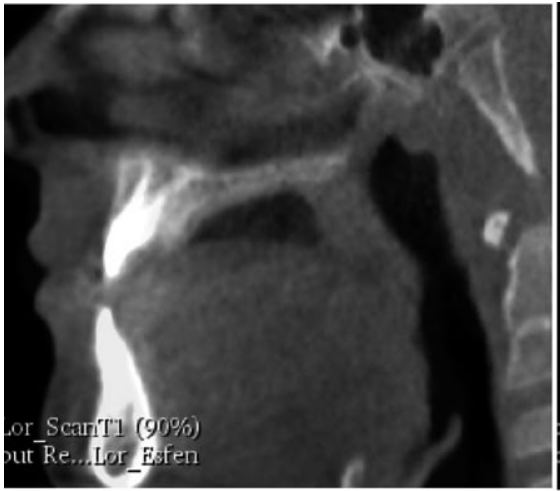

T1

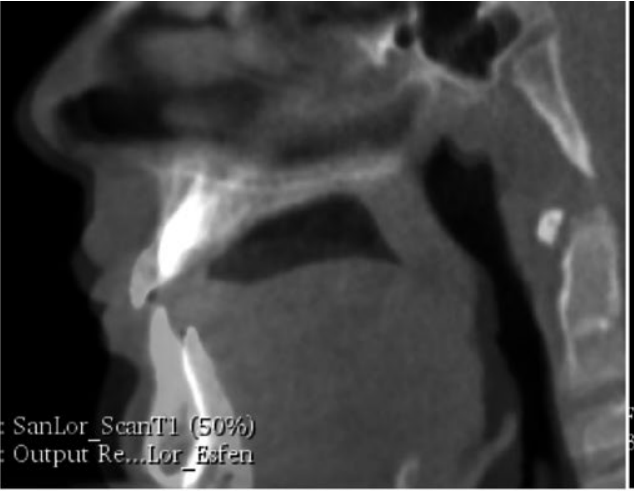

superimposition $\mathrm{T} 1 / \mathrm{T} 2$

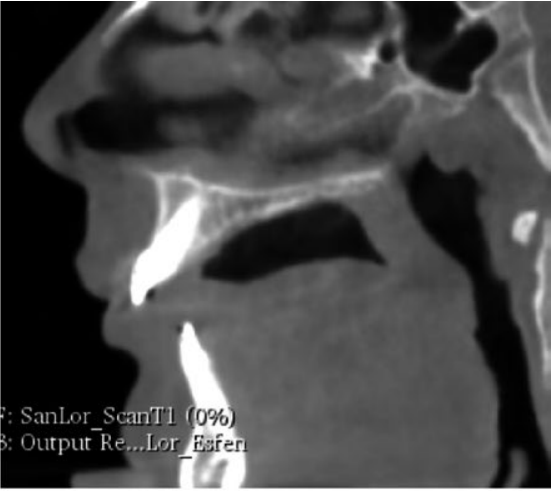

$\mathrm{T} 2$

FIGURE 4 Images of before (T1) and after therapy (T2) scans displaying upper incisors eruption during the interval between both scans. The vertical displacement of the upper permanent central incisor inside the alveolar bone may influence changes in Point $A$. When the crown of the incisor was at the level of the point A, Point A appeared more prominent anteriorly than when the central incisor had erupted previously (and the root was at the level of Point $A$ )

\section{5 | CONCLUSIONS}

- The Alt-RAMEC/FM and RME/FM protocols are effective treatment protocols for correction of Class III malocclusion in patients in the deciduous/early mixed dentition.

- The Alt-RAMEC/FM and RME/FM protocols produced similar skeletal and dental effects, with neither group providing a more favourable outcome.

- Skeletal effects of the protraction, as measured at ANS and infrazygomatic midpoint, resulted in 50\% forward (A-P) and 50\% downward (S-I) movement.

- Despite the use of a bonded expander, both groups exhibited more forward and downward dental movement, as measured at the primary canine, when compared to the movement measured at the skeletal landmarks.

\section{ORCID}

L. Franchi iD http://orcid.org/0000-0002-2072-460X

\section{REFERENCES}

1. Hardy DK, Cubas YP, Orellana MF. Prevalence of angle class III malocclusion: a systematic review and meta-analysis. Open J Epidemiol. 2012;2:75-82.

2. McNamara JA Jr, Brudon WL. Orthodontics and Dentofacial Orthopedics. Ann Arbor, Michigan: Needham Press; 2001:375-385.

3. Foersch $M$, Jacobs $C$, Wriedt $S$, Hechtner $M$, Wehrbein $H$. Effectiveness of maxillary protraction using facemask with or without maxillary expansion: a systematic review and meta-analysis. Clin Oral Investig. 2015;19:1181-1192.

4. Cordasco G, Matarese G, Rustico L, et al. Efficacy of orthopedic treatment with protraction facemask on skeletal Class III malocclusion: a systematic review and meta-analysis. Orthod Craniofac Res. 2014;17:133-143
5. Woon SC, Thiruvenkatachari B. Early orthodontic treatment for Class III malocclusion: a systematic review and meta-analysis. Am J Orthod Dentofacial Orthop. 2017;151:28-52.

6. Rongo R, D'Antò V, Bucci R, Polito I, Martina R, Michelotti A. Skeletal and dental effects of Class III orthopaedic treatment: a systematic review and meta-analysis. J Oral Rehabil. 2017;44:545-562.

7. Liou EJ-W. Effective maxillary orthopedic protraction for growing Class III patients: a clinical application simulates distraction osteogenesis. Prog Orthod. 2005;6:154-171.

8. Liou EJ-W. Toothborne orthopedic maxillary protraction in Class III patients. J Clin Orthod. 2005;39:68-75.

9. Do-deLatour TB, Ngan P, Martin CA, Razmus T, Gunel E. Effect of alternate maxillary expansion and contraction on protraction of the maxilla: a pilot study. Hong Kong Dent J. 2009;6:72-82.

10. Isci D, Turk T, Elekdag-Turk S. Activation-deactivation rapid palatal expansion and reverse headgear in Class III cases. Eur J Orthod. 2010;32:706-715.

11. Masucci C, Franchi L, Giuntini V, Defraia E. Short-term effects of a modified Alt-RAMEC protocol for early treatment of Class III malocclusion: a controlled study. Orthod Craniofac Res. 2014;17:259-269.

12. Liu W, Zhou Y, Wang X, Liu D, Zhou S. Effect of maxillary protraction with alternating rapid palatal expansion and constriction vs expansion alone in maxillary retrusive patients: a single-center, randomized controlled trial. Am J Orthod Dentofacial Orthop. 2015;148:641-651.

13. Pithon MM, Santos NL, Santos CR, et al. Is alternate rapid maxillary expansion and constriction an effective protocol in the treatment of Class III malocclusion? A systematic review. Dental Press J Orthod. 2016;21:34-42.

14. Baccetti T, Franchi L, McNamara JA Jr. The cervical vertebral maturation (CVM) method for the assessment of optimal treatment timing in dentofacial orthopedics. Semin Orthod. 2005;11:119-129.

15. McNamara JA Jr, Franchi L. The cervical vertebral maturation method: a user's guide. Angle Orthod. 2018;88:133-143.

16. Ngan P, Hagg U, Yiu C, Wei SH. Treatment response and long-term dentofacial adaptations to maxillary expansion and protraction. Semin Orthod. 1997;3:255-264.

17. Ruellas AC, Tonello C, Gomes LR, et al. Common 3-dimensional coordinate system for assessment of direction changes. Am J Orthod Dentofacial Orthop. 2016;149:645-656.

18. Ruellas AC, Huanca Ghislanzoni LT, Gomes MR, et al. Comparison and reproducibility of 2 regions of reference for maxillary regional 
registration with cone-bean computed tomography. Am J Orthod Dentofacial Orthop. 2016;149:533-542.

19. Springate SD. The effect of sample size and bias on the reliability of estimates of error: a comparative study of Dahlberg's formula. Eur J Orthod. 2012;34:158-163.

20. Fleiss JL. The design and analysis of clinical experiments. New York, NY: John Wiley and Sons, Inc.; 1986:7.

21. Fedorov A, Beichel R, Kalpathy-Cramer J, et al. 3D slicer as an image computing platform for the quantitative imaging network. Magn Reson Imaging. 2012;30:1323-1341.

22. Melsen B, Melsen F. The postnatal development of the palatomaxillary region studied on human autopsy material. Am J Orthod. 1982;82:329-342.

\section{SUPPORTING INFORMATION}

Additional supporting information may be found online in the Supporting Information section at the end of the article.

How to cite this article: Fischer B, Masucci C, Ruellas A, et al. Three-dimensional evaluation of the maxillary effects of two orthopaedic protocols for the treatment of Class III malocclusion: A prospective study. Orthod Craniofac Res. 2018;21:248-257. https://doi.org/10.1111/ocr.12247 